\title{
Near-infrared evidence for a sudden temperature increase in Eta Carinae ${ }^{\star}$
}

\author{
Andrea Mehner ${ }^{1}$, Kazunori Ishibashi ${ }^{2}$, Patricia Whitelock ${ }^{3,4}$, Takahiro Nagayama ${ }^{2}$, Michael Feast ${ }^{3,4}$, \\ Francois van $\mathrm{Wyk}^{3}$, and Willem-Jan de $\mathrm{Wit}^{1}$
}

\author{
1 ESO, Alonso de Cordova 3107, Casilla 19001, Vitacura, Santiago de Chile, Chile \\ e-mail: amehner@eso.org \\ 2 Division of Elementary Particle Physics and Astrophysics, Graduate School of Science, Nagoya University, 464-8602 Nagoya, \\ Japan \\ 3 South African Astronomical Observatory, PO Box 9, Observatory, 7935 Cape Town, South Africa \\ 4 Astronomy, Cosmology and Gravity Centre, Astronomy Department, University of Cape Town, 7701 Rondebosch, South Africa
}

Received 22 September 2013 / Accepted 20 January 2014

\begin{abstract}
Aims. Eta Car's ultra-violet, optical, and X-ray light curves and its spectrum suggest a physical change in its stellar wind over the last decade. It has been proposed that the mass-loss rate has decreased by a factor of about 2 over the last 15 years. We complement these recent results by investigating the past evolution and the current state of $\eta$ Car in the near-infrared (IR).

Methods. We present JHKL photometry of $\eta$ Car obtained at SAAO Sutherland from 2004-2013 with the Mk II photometer at the $0.75 \mathrm{~m}$ telescope and $J H K_{\mathrm{S}}$ photometry with SIRIUS at the $1.4 \mathrm{~m}$ IRSF telescope from 2012-2013. The near-IR light curves since 1972 are analyzed.

Results. The long-term brightening trends in $\eta$ Car's JHKL light curves were discontinuous around the 1998 periastron passage. After 1998 , the star shows excess emission above the extrapolated trend from earlier dates, especially in $J$ and $H$, and the blueward, cyclical progression in its near-IR colors is accelerated. The near-IR color evolution is strongly correlated with the periastron passages. After correcting for the secular trend we find that the color evolution matches an apparent increase in blackbody temperature of an optically thick near-IR emitting plasma component from about $3500 \mathrm{~K}$ to $6000 \mathrm{~K}$ over the last 20 years.

Conclusions. We suggest that the changing near-IR emission may be caused by variability in optically thick bremsstrahlung emission. Periastron passages play an important role in the observed excess near-IR emission after 1998 and the long-term color evolution. We thus propose the hypothesis that angular momentum transfer (via tidal acceleration) during periastron passages leads to sudden changes in $\eta$ Car's atmosphere resulting in a long-term decrease in the mass-loss rate.
\end{abstract}

Key words. stars: massive - stars: variables: S Doradus - stars: individual: $\eta$ Carinae - stars: winds, outflows - stars: mass-loss

\section{Introduction}

Eta Car is, with $M>100 M_{\odot}$ and $L \sim 5 \times 10^{6} L_{\odot}$, one of the most massive and most luminous stars in our Galaxy. During its Great Eruption almost two centuries ago, its luminous energy output rivaled that of a supernova (SN) as it expelled more than $10 M_{\odot}$ (Smith et al. 2003), which is now observed as the Homunculus nebula. Because of its proximity to us, $\eta$ Car is one of a few SN impostors whose recovery and continuing instability can be analyzed in detail. Diverse datasets cover the last four centuries, but despite numerous multi-wavelength studies there is still no consensus on the nature of $\eta$ Car and the cause of its Great Eruption (Davidson \& Humphreys 1997; 2012, Morse et al. 1999; Humphreys \& Stanek 2005; and references therein). It is now generally believed that $\eta$ Car is a binary system (Damineli 1996; Damineli et al. 2000; cf. Davidson 1997; Davidson et al. 2000). However, the details of the orbital parameters and the companion's influence on the observables are still debated. For recent discussions, see Groh et al. (2012a),

* Tables 1 and 2 are only available at the CDS via anonymous ftp to cdsarc.u-strasbg.fr (130.79.128.5) or via

http://cdsarc.u-strasbg.fr/viz-bin/qcat?J/A+A/564/A14
Madura et al. (2012b), Okazaki et al. (2008), and for an alternative model Kashi \& Soker (2008b).

Most of the flux from the $\eta$ Car system emerges at midto far-infrared (IR) wavelengths and is reprocessed shortwavelength radiation from the central stars by cool dust in the Homunculus nebula (e.g., Smith et al. 2003, and references therein). At optical wavelengths we observe the light originating from $\eta$ Car's stellar wind, the Weigelt knots and similar close ejecta, and dust-scattered light from the Homunculus nebula. The angular size of the system is smallest at near-IR wavelengths and near-IR photometry is therefore particularly valuable for ground-based monitoring of the central source. Images reach a minimum size around $2 \mu \mathrm{m}$ (Gehrz et al. 1973; Allen 1989), but cyclical variations in the spatial extent exist. Smith \& Gehrz (2000) found that the central core changed its morphology in the $K$ band between 1995 and 1998, appearing more pointlike close to periastron in 1998. In this paper we discuss the near-IR light curves obtained from large aperture photometry of the entire $\eta$ Car system. The near-IR radiation arises from more than one component, but is likely dominated by free-free emission from the stellar wind (Whitelock et al. 2004; Whitelock 2005). Other sources include scattered light from the Homunculus and 
from close ejecta like the Weigelt knots (Weigelt \& Ebersberger 1986; Davidson et al. 1995; Weigelt \& Kraus 2012), emission lines $(<10 \%$, mostly $\mathrm{HI}$ and $\mathrm{He} \mathrm{I})$, and at the $K$ and $L$ bands hot circumstellar dust ( $T_{\text {dust }} \gg 200 \mathrm{~K}$ ) contributes. Conceivably all components play a role, but the importance of each source is still debated. The interpretation of data on $\eta$ Car is difficult because different types of observation refer to physically separate and distinct regions and may experience different extinctions (Davidson et al. 1995).

The near-IR light curves show variations on different timescales: secular brightening and color evolution, quasiperiodic variations associated with the $5.5 \mathrm{yr}$ orbit of the companion star, and variations on timescales of a few weeks to months. Observed variations are likely to be a combination of several processes, such as the decreasing extinction with the thinning of the expanding Homunculus nebula, dispersion and possibly accretion of circumstellar and wind material by the companion star, varying dust production and destruction, and changes in the stellar wind (e.g., altered stellar rotation and mass-loss rate, a wind cavity around the companion, and altered photoionization of the primary wind due to the companion).

The gradual brightening of $\eta$ Car in the visual and near-IR since the 1940s is generally attributed to a gradual decrease in extinction as the dust formed in the Great Eruption disperses (e.g., Davidson 1971; van Genderen et al. 1994). The rate of brightening was about the same in the visual and near-IR and the dust grains were therefore thought to be predominantly large producing near-neutral extinction. However, the accelerated rate of brightening after 1997, especially in the ultra-violet (UV), is incompatible with a simple model of decreasing extinction as a result of the steady expansion of a dust shell (Davidson et al. 1999, 2005; Sterken et al. 1999; van Genderen \& Sterken 2004). Whitelock et al. (2004) noted that the near-IR brightening was faster and that the color changes have been greater for the two cycles in 1992.5-2003.5 than for the two previous ones. The simplest interpretation of $\eta$ Car's recent brightening at UV to near-IR wavelengths, its altered X-ray light curve, and the major spectral changes observed in the stellar wind features is a decrease in its mass-loss rate (Kashi \& Soker 2009a; Corcoran et al. 2010; Mehner et al. 2010, 2012). However, conceivably these observations could be accounted for by line-of-sight effects (Groh et al. 2012a,b; Madura et al. 2013). A scenario can be envisioned in which we previously looked down the wall of the wind-wind cavity with a large column density. A small change in the stellar parameters could have changed the wind cone opening angle, resulting in lower column density along our line of sight.

In this paper we investigate $\eta$ Car's current state in the nearIR to complement the recent work in other wavelength regions. Eta Car has been monitored in JHKL at the South African Astronomical Observatory (SAAO) at Sutherland for more than 40 years starting in 1972 (Whitelock et al. 1983; 1994, Feast et al. 2001; Whitelock et al. 2004). We present the most recent JHKL photometry obtained with the Mk II photometer at the $0.75 \mathrm{~m}$ telescope between 2004-2013. The Mk II photometry is the most consistent dataset of $\eta$ Car, spanning a timebaseline of several decades and is thus uniquely suited to analyze secular changes of this stellar system. However, there are plans to close down the $0.75 \mathrm{~m}$ telescope and a temporal overlap of observations with another system is essential to assure the continuation of these legacy observations. Thus, in 2012, we started a $J H K_{\mathrm{s}}$ monitoring program with the Simultaneous3color InfraRed Imager for Unbiased Survey (SIRIUS) at the InfraRed Survey Facility (IRSF) at SAAO.
In this paper we stress the importance of the binary nature of $\eta$ Car on the observables. The long-term evolution of $\eta$ Car's light curve and colors may be especially a result of changes in the stellar system (either the stars themselves or the surrounding material) triggered at periastron passages. Whitelock et al. (2004) already noted that the 1998 periastron was a phase of rapid change and that the near-IR colors change discontinuously at periastron passages and thus may be driven by some physical process associated with the orbital period. In Sect. 2 we describe the observations and data analysis. In Sect. 3 we present our results, followed by a discussion in Sect. 4 , and the conclusion in Sect. 5.

\section{Observations and data analysis}

\subsection{JHKL photometry with the Mk II photometer}

A near-IR monitoring campaign of $\eta$ Car has been in progress at SAAO since 1972 (Whitelock et al. 1983, 1994, 2004; Feast et al. 2001). Observations were carried out regularly with the Mk II photometer using a 36" aperture encompassing the entire Homunculus nebula, which has an extension of about $20^{\prime \prime}$. Observations from 1972 to 1973 were conducted at the 18 -in, the $1-\mathrm{m}$, and the 1.9-m telescopes. Subsequent observations were carried out at the $0.75 \mathrm{~m}$ telescope (the detector was changed only once in 1978 April). The very long time-baseline of more than 40 years of consistent observations makes this dataset invaluable.

The Mk II photometry from the years 1972-2004 was published in Whitelock et al. (1983, 1994); Feast et al. 2001), and Whitelock et al. (2004). The SAAO IR standard system (Carter 1990) was used and uncertainties range from about 0.03 mag in $J H K$ to $0.05 \mathrm{mag}$ in $L$. Here we present the most recent Mk II photometry from 2004-2013 (Table 1). For details about the observations and data reduction, see the papers cited above.

\section{2. $J H K_{\mathrm{S}}$ photometry with SIRIUS}

In 2012 May we started a near-IR monitoring campaign of $\eta$ Car with the SIRIUS camera at the $1.4 \mathrm{~m}$ IRSF telescope at SAAO (Glass \& Nagata 2000) ${ }^{1}$. SIRIUS is a simultaneous threechannel $J H K_{\mathrm{s}}$ camera with a field of view of $7.7^{\prime} \times 7.7^{\prime}$ and a pixel scale of $0.45^{\prime \prime} /$ pixel (Nagashima et al. 1999; Nagayama et al. 2003). The data include several hours of consecutive observations to explore the potential presence of hourly to daily variations, which we have not identified. We note, however, that we are only sensitive in our data analysis to variation larger than $\sim 0.01 \mathrm{mag}$. Simultaneous observations with the Mk II photometer were performed to determine the transformation between the two photometric systems.

Each observation consisted of ten dithered exposures to correct for bad pixels. A nearby field with few stars was observed within $30 \mathrm{~min}$ of the science exposures to create a sky image. Observations had to be carried out with neutral density filters to avoid overexposure. This made it impossible to determine $\eta$ Car's magnitudes based on relative photometry to other stars in the field of view. Instead, we determined the extinction coefficients and zero points from standard stars that were observed each night with airmasses ranging from 1.0 to 2.4. To facilitate the comparison with the Mk II photometry we chose two standards (BS4450 and BS4382) from the same list of standards

1 IRSF is a joint project of Nagoya University, Kyoto University, the National Astronomical Observatory of Japan (NAOJ), and SAAO. 


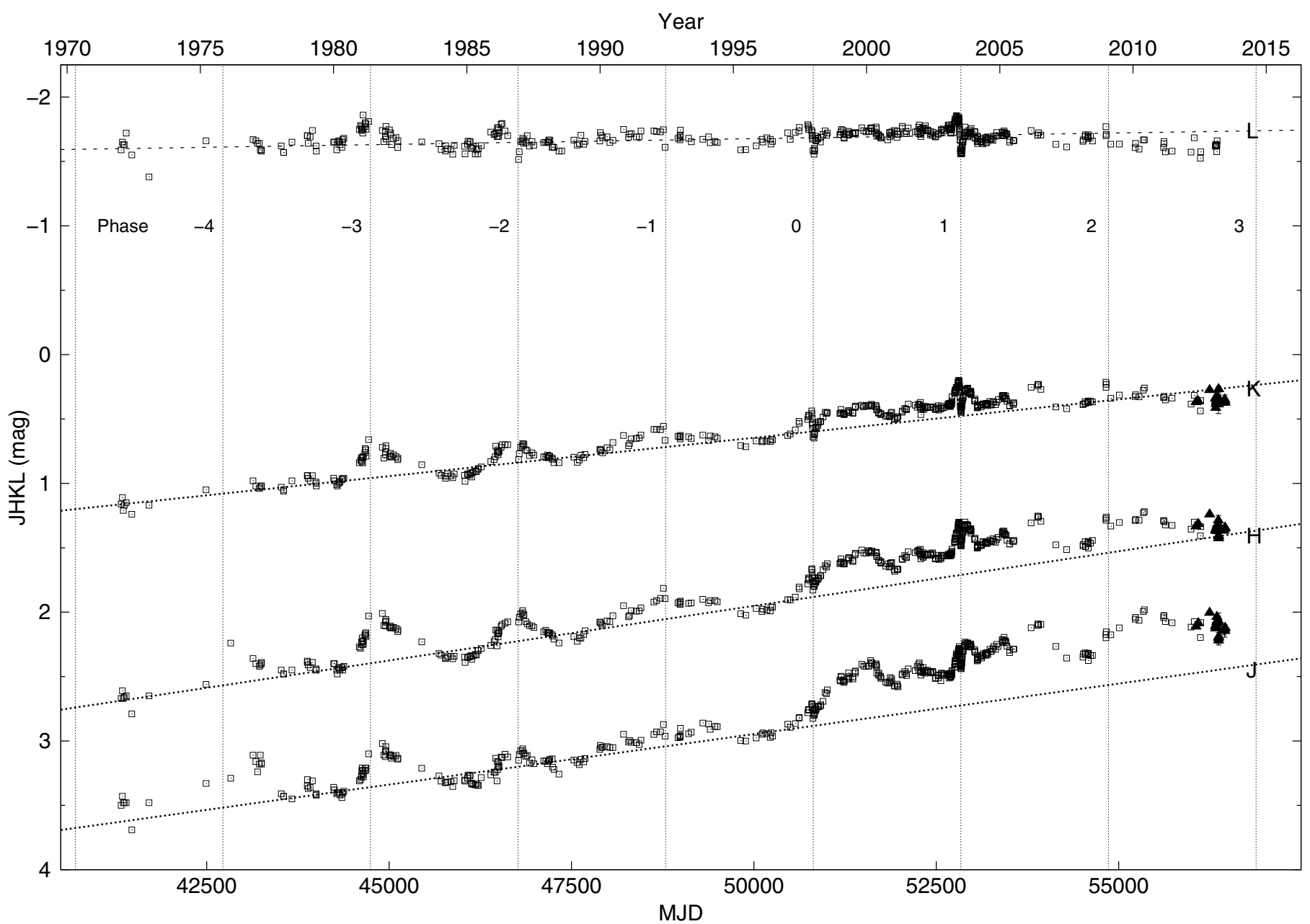

Fig. 1. JHKL light curves from 1972-2013 (open squares: Mk II, filled triangles: SIRIUS). The errors at $J H K$ are less than \pm 0.03 mag, while those at $L$ are about \pm 0.05 mag. Dotted lines show linear regression trends based on the pre-1998 data. Vertical dotted lines indicate periastron passages (the corresponding phases are indicated). In $J, H$, and to a lesser degree in $K$ and $L, \eta$ Car did not follow the long-term linear brightening trends after the 1998 periastron passage.

(Carter 1990) and with similar near-IR magnitudes to $\eta$ Car. None of the standard stars has $\eta$ Car's color.

The data were reduced using the external IRAF package SIRIUS (v.09). Master dark frames were created and subtracted from the science frames. For the flat field response we created differential images of twilight frames without neutral density filters (to reach reasonable count levels), which were mediannormalized and median-combined. Eta Car and the standard stars were always centered on the same pixel areas and any vignetting or pattern introduced by the neutral density filters are thus not significant for the resulting photometry. We mitigated the effect of an inherent vertical discontinuity in the bias count levels across the array boundaries to the flat field response image by rejecting some differential images that inherited the central vertical step. Sky frames were subtracted from each science frame and the dithered exposures were re-centered and combined.

The photometry was carried out with standard IRAF tools. We chose an aperture of $36^{\prime \prime}$ consistent with the analysis performed by Whitelock et al. (1983, 1994), Whitelock et al. 1994, Feast et al. 2001, and Whitelock et al. (2004). To compare the SIRIUS and the Mk II photometry we explored two different methods. First, we determined $\eta$ Car's $J H K_{\mathrm{s}}$ magnitudes in the SIRIUS system and converted them to the Carter system. There is no published direct transformation formula between the
SIRIUS and Carter photometric systems and we thus performed a series of transformations. Second, in 2013 February we conducted simultaneous observations with SIRIUS and Mk II and derived a linear transformation for $\eta$ Car between the two systems $\left(J_{\text {Mk II }} \approx J_{\text {SIRIUS }}+0.101 \mathrm{mag}, H_{\text {Mk II }} \approx H_{\text {SIRIUS }}-0.047 \mathrm{mag}\right.$, $\left.K_{\text {Mk II }} \approx K_{\text {SIRIUS }}-0.076 \mathrm{mag}\right)$. The first method resulted in very good results for $J$ and $H$, but we systematically underestimated the resulting $K$ magnitude by $0.04 \mathrm{mag}$. We thus adopted the linear transformation obtained from the simultaneous observations. We note, however, that $\eta$ Car's color is changing (secular and cyclical changes) and that this transformation will need to be revised in the future. The SIRIUS photometry from 2012-2013 can be found in Table 2 .

\section{Results}

\subsection{The JHKL light curves 1972-2013}

Eta Car has been monitored for more than 40 years in $J H K L$ at SAAO. Until 1998 the light curves showed an approximately linear brightening trend, superimposed with quasi-periodic variations (see Fig. 1). The overlying quasi-periodic variations associated with the $5.5 \mathrm{yr}$ orbit of the companion star render it difficult to determine the rate and confirm the cause of this secular brightening. We determined linear regression lines by selecting only data points obtained at the $0.75 \mathrm{~m}$ telescope between 1973 


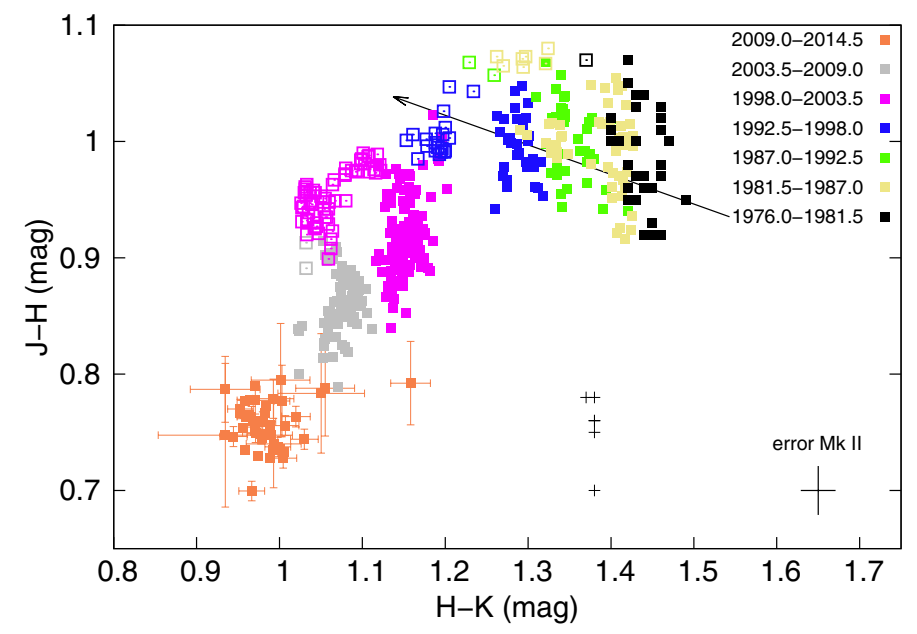

Fig. 2. $J-H$ versus $H-K$ color-color diagram from 1976-2013. The key refers to the time windows of orbital cycles and not to the actual observation dates. Each cycle is displayed with a different color code to visualize the long-term color evolution and the impact of the periastron passages. A cycle here consists of phases $0.05-1.05,1.05-2.05$, etc., to include only one periastron passage per cycle. Open squares of the same color show data points close to periastron $(t=-40$ days to $t=$ +100 days). The arrow indicates the expected evolution from 19702014 based on the pre-1998 data. The data points represented by the black plus symbols are from observations obtained before 1978 with a different detector.

and 1998 at phases $0.50-0.85$ in the $5.5 \mathrm{yr} \mathrm{cycle}^{2}$. This phase interval was chosen because of the minimal influence of the companion star during the epoch around apastron. In addition, the near-IR excess observed at periastron subsides around phase 0.5 . We find that the star brightened by $\Delta J=0.029 \pm 0.001 \mathrm{mag} \mathrm{yr}^{-1}$, $\Delta H=0.031 \pm 0.001 \mathrm{mag} \mathrm{yr}^{-1}, \Delta K=0.022 \pm 0.001 \mathrm{mag} \mathrm{yr}^{-1}$,

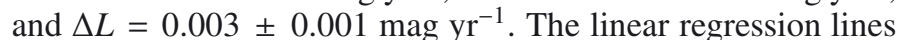
are shown in Fig. 1 to visualize this long-term brightening trend.

A strong discontinuity in the long-term near-IR brightening trend is observed around the 1998 periastron passage. The system suddenly showed accelerated brightening, most pronounced in $J$ and $H$. For the last 15 years, the light curve in $J$ has shown an average excess emission of about 0.35 mag above the extrapolation from the previous trend. The light curve in $H$ had recovered by 2013 to the brightness expected from the long-term trend. In $K$ and $L, \eta$ Car showed little or no excess emission and in 2013 the star became fainter in both wavebands than expected from the previous trends. The light curves alone do not reveal whether the secular brightness increase and the break in 1998 are related to processes occurring at periastron, yet it is suggestive that the 1998 discontinuity coincides with a periastron passage.

\subsection{Long-term and cyclical color variations}

Eta Car's near-IR color evolution reveals a strong discontinuity around the 1998 periastron passage. Figure 2 shows a color-color diagram $(J-H$ versus $H-K)$ from 1976-2013, covering seven orbital periods. Earlier observations are not included in the following discussion for consistency reasons (they were obtained at different telescopes). The outlying data points

\footnotetext{
2 When referring to "phase" in the $5.5 \mathrm{yr}$ cycle, we use a period of 2023.0 days; see the appendix in Mehner et al. (2011). We denote time close to periastron passage by $t$, such that $t=0$ at MJD 54860.0 (2009 January 29), MJD 52837.0 (2003 July 17), etc. Periastron most likely occurs within the range $t \approx-15$ to +15 days.
}

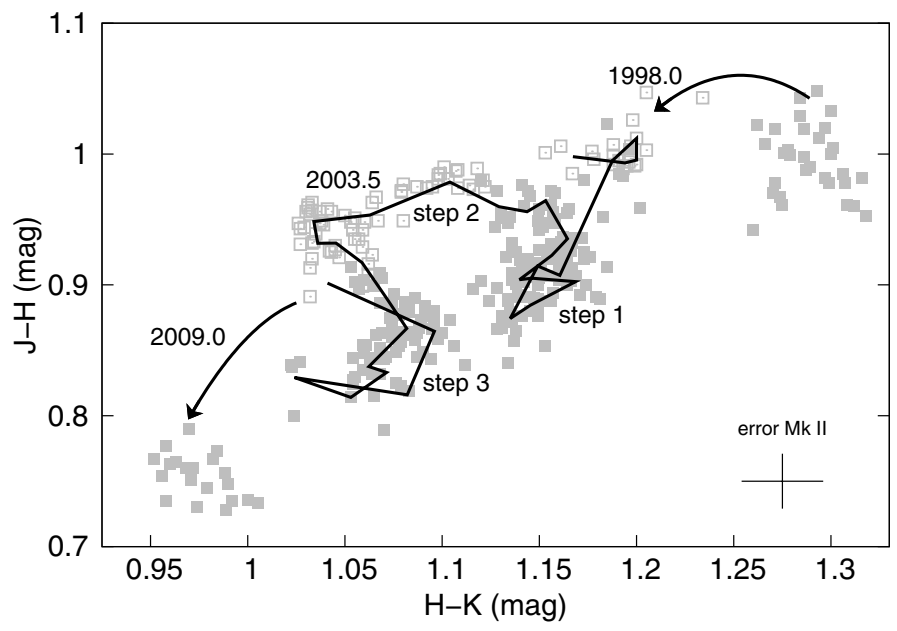

Fig. 3. $J-H$ versus $H-K$ color-color diagram. Filled squares are observations outside periastron passages (step 1: phases $0.05-0.98$, step 3: phases 1.05-1.98). Open squares are observations during periastron (step 2: phases $0.98-1.05$, i.e., 2003.5 periastron). The solid curve highlights the quasi-cyclical cycloidal pattern.

of cycle 1976-1981.5 (the black plus symbols in Fig. 2) are from observations obtained before 1978 with the previous detector (a lead sulphide detector). The change to the indium antimonide detector still in use did not alter significantly the $H K L$ magnitudes obtained, but $J$ became fainter by $\sim 0.20$ mag (Whitelock et al. 1983). A possible reason is that the sensitivity of the old detector may have been greater at the short wavelength end of the $J$ band and thus may have been more sensitive to the He I $10830 \AA$ emission line. The data points are presented here to show how relevant the question of detectors/filters is when combining different data sets.

Between 1976 and 1998, $J-H$ was almost constant with

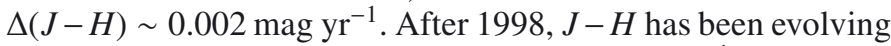

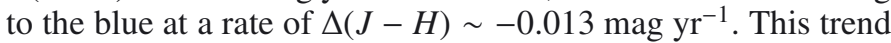
appears to be primarily induced at the periastron passages starting in 1998 . The $J-H$ color varies by about 0.1 mag throughout the $5.5 \mathrm{yr}$ cycle, but shows a sudden increment on the order of 0.1 mag towards the blue during periastron. Since 1976 $H-K$ had been slowly evolving towards the blue at a rate of $\Delta(H-K) \sim-0.009$ mag $\mathrm{yr}^{-1}$. After $1998, H-K$ is evolving

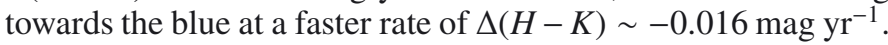
As in the case of $J-H$, the blueward evolution of $H-K$ appears to be mostly triggered at periastron passages; a fast change (on a timescale of a few weeks) to the blue at each periastron is evident in Fig. 2 (see also Figs. 3 and 4 from Whitelock et al. 2004).

The long-term color evolution (filled squares in Fig. 2) is interspersed by a cyclical pattern. A cycloidal, counter-clockwise $5.5 \mathrm{yr}$ pattern is outlined in the color-color diagram (see Fig. 3). Shortly after periastron, $J-H$ and $H-K$ vary gradually over the next few years (step 1 in Fig. 3). The $J-H$ value decreases to a minimum before becoming redder again. The movement to the red is not in the middle of the cycle, but about $1 \mathrm{yr}$ before the next periastron. During this epoch, $H-K$ scatters within about 0.05 mag but shows little directed evolution. At the end of a cycle, $J-H$ reaches its reddest value of that cycle again. Then, within 3-4 weeks and around periastron, $H-K$ evolves about 0.1 mag to the blue at almost constant $J-H$ (step 2). The next cycle starts and $J-H$ becomes bluer (step 3, which corresponds to the same stage in the cycle as step 1). For orbital cycles prior 


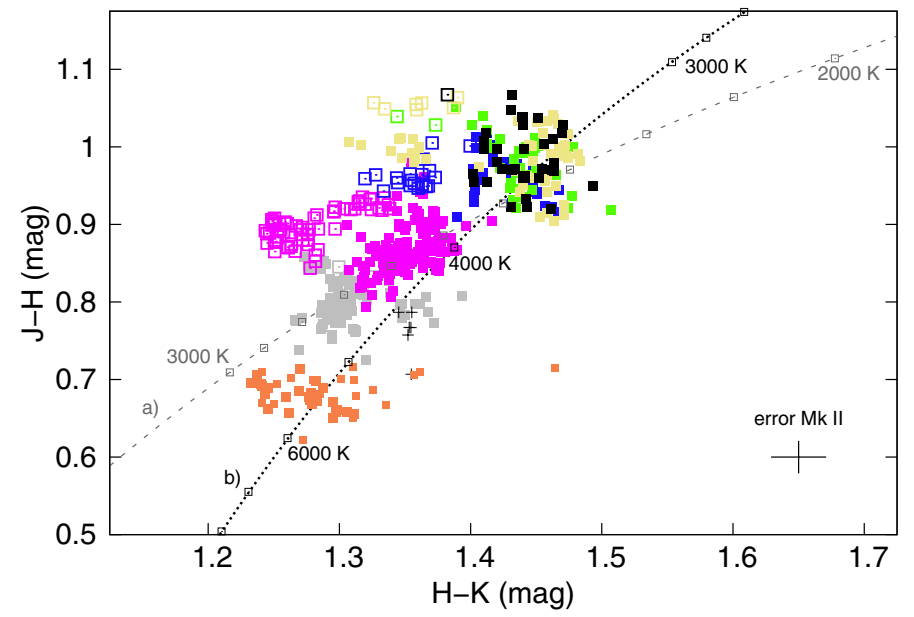

Fig. 4. $J-H$ versus $H-K$ color-color diagram as in Fig. 2 (the same symbols are used), but corrected for the secular brightening. The dashed gray curve a) traces the expected colors of a blackbody reddened by $E(J-H)=0$ mag and $E(H-K)=0.863$ mag; the dotted black curve b) shows a blackbody reddened by $E(J-H)=0.4$ mag and $E(H-K)=$ $1.2 \mathrm{mag}$ (see text). The temperature steps, indicated with open squares along the blackbody functions, are $100 \mathrm{~K}$ between $2000-3000 \mathrm{~K}$ and $1000 \mathrm{~K}$ for higher temperatures.

to $1992-1998$ this pattern traces a horizontal cycloidal structure in the color-color diagram $(\Delta(J-H) \approx$ const. $)$, while for later cycles there is also a vertical trend $(\Delta(J-H)<0)$.

Under the assumption that the long-term linear brightening trend before 1998 is due to the dissipation of dust as the Homunculus nebula expands, we correct the light curves accordingly to obtain the near-IR excess emission. For simplicity, we do not consider a potential non-linear brightening trend. Figure 4 shows the same color-color diagram as Fig. 2, but corrected for the long-term (linear) trend. The filled squares show the data points outside periastron passages and represent $\eta$ Car's longterm color evolution. The open squares show the data points close to periastron, which lie in a separate region in color-color space.

Until 1998, the data points fall within the same part of color-color space, but starting around the 1998 periastron passage a long-term blueward color evolution sets in. In the following we ignore the cyclical variations (especially step 2 in Fig. 3). They are probably caused by photoionization of the primary wind by UV radiation of the companion star (or possibly the primary star itself) and/or a wind cavity around it. Instead, we focus on the long-term blueward color evolution, which resembles a change in blackbody temperature. This suggests that we observe a change in a significant contribution from an optically thick near-IR emitting plasma radiating nearly at blackbody temperature. Blackbody temperature functions for two different sets of extinction values are displayed in Fig. 4. The extinction towards $\eta$ Car is uncertain, but because of the similar long-term trends in $J$ and $H$ we assume a minimum value for $E(J-H)$ of 0 mag. A reddening of $E(J-H) \approx 0$ mag and $E(H-K)=0.863 \pm 0.003 \mathrm{mag}$ then fits $\eta$ Car's data points (curve a in Fig. 4). In this case the blueward trend of $\eta$ Car from 1998 to 2013 resembles an apparent blackbody temperature change from $2400 \mathrm{~K}$ to $3000 \mathrm{~K}$. We note that this is a lower limit in the case that a negligible amount or no optically thin plasma is involved in the emission process. In addition, we use a simple regression test to search for the set of extinction values that best describes the blueward trend (including

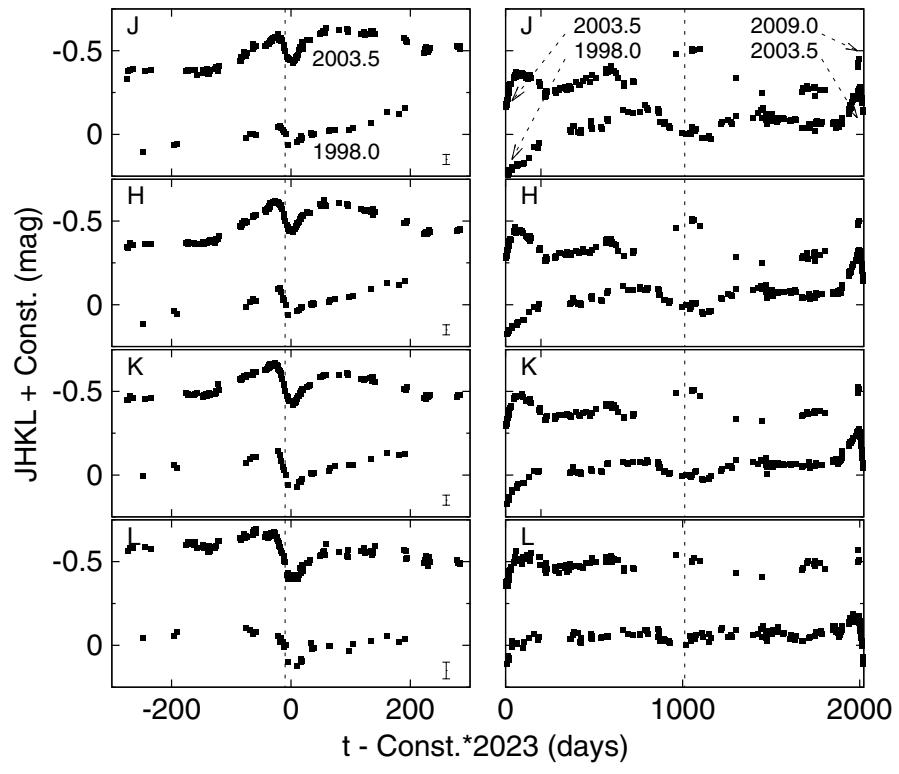

Fig. 5. JHKL light curves showing the cycle-to-cycle differences of the two cycles between 1998.0 and 2009.0. The light curves were not corrected for the linear brightening trend. The vertical lines indicate the mid-decline in the light curve during the 1998 periastron passage (left panels, typical error bars are displayed in the lower right corner) and apastron (right panels).

only phases $0.5-0.85$ to minimize the influence of step 2 in Fig. 3). We find a reddening of $E(J-H)=0.4 \pm 0.1 \mathrm{mag}$ and $E(H-K)=1.2 \pm 0.1 \mathrm{mag}^{3}$. In this case, $\eta$ Car's blueward trend resembles an apparent blackbody temperature change from $3500 \mathrm{~K}$ to $6000 \mathrm{~K}$ (curve b in Fig. 4).

As already noted by Whitelock et al. (2004), periastron passages appear to play an important role in the color evolution of $\eta$ Car. The long-term blueward evolution in $H-K$ and, after 1998 , also in $J-H$ occurs in discontinuous steps close to periastron. The 1998 periastron passage may have been particularly important leading to a significant change in the system that can be observed in the near-IR light curves and color evolution. The $5.5 \mathrm{yr}$ quasi-periodic variations make it difficult to determine whether the apparent secular color trends are constant or occur in discontinuous episodes close to periastron. However, the involved timescales of only a few weeks support the idea that during periastron permanent changes are induced in the system colors, i.e., the system does not recover to its out-of-periastron state. This observational evidence suggest that the system is changed by the periastron events.

\subsection{Cycle-to-cycle variations}

The JHKL light curves show quasi-periodic variations of $5.5 \mathrm{yrs}$ associated with the orbit of the companion star. Figure 5 compares the light curves during the periastron passages in 1998.0 and 2003.5 and the mid-cycle light curves from the two cycles between 1998.0 and 2009.0. Variations occur simultaneously in all four bands, but significant cycle-to-cycle differences exist.

Excess emission is observed for about 1 year around the periastron passages only interrupted by a strong decline of a few weeks around $t=0$ days. The light curves leading up to periastron are similar for each cycle; the brightness increases in

3 The quoted errors are systematic errors reflecting the spread of the selected data points around the blueward trend. 
all bands followed by a strong decline to a minimum. The recovery after the decline, however, differs from cycle-to-cycle. For example, in 1998, the recovery was slow and almost linear, while in 2003.5, on the same timescale, the light curve quickly reached another maximum and decreased again. At apastron, when the influence of the companion star is minimal, the light curves also differ considerably from cycle-to-cycle. During the 2000 apastron there was a local minimum in $\eta$ Car's near-IR brightness, while 5.5 yrs later during the next apastron in 2006 the light curves showed a local maximum.

Unfortunately, only the 1998.0 and the 2003.5 periastron passages and a few mid-cycles are well-sampled in the near-IR. Nevertheless, together with the less frequent observations in other cycles, it is clear that the light curves vary from cycle to cycle making it difficult to fit one single model to the data. Most likely different processes, such as photoionization of the primary wind and a wind cavity, contribute in varying degrees.

\section{Discussion}

The JHKL light curves from 1972 to 1998 show an almost linear increase in brightness, which can be explained with a simple model of dust dispersion as the Homunculus nebula expands (e.g., Davidson 1971; van Genderen et al. 1994). We find that prior to $1998 \eta$ Car brightened at a rate of about $0.022-0.031 \mathrm{mag}^{-1}$ in $J H K$, similar to its brightening rate in the visual. Van Genderen et al. (1994) found that the star brightened almost linearly in $V$ between 1952-1992 at a rate of

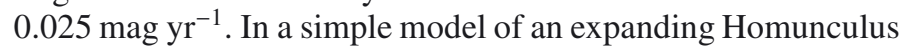
nebula, this near-neutral extinction implies large dust grains. The

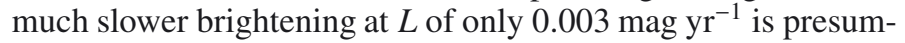
ably due to the reduced effects of extinction and a stronger contribution by thermal dust emission from the Homunculus (Smith 2010).

The accelerated brightening in the UV to near-IR wavelengths after 1998 is inconsistent with this simple model (e.g., Davidson et al. 1999; Sterken et al. 1999, 2005). Van Genderen et al. (2006) and Whitelock et al. (2004) noted that the accelerated brightening may be related to the 1998 periastron passage. Because $\eta$ Car is close to the Eddington limit, it is unlikely that the observations can be accounted for by an intrinsic brightening of the star. Davidson et al. (1999) argued that the hot dust near the star (at a distance of a few hundred AU) must have decreased. Either existing dust was destroyed and/or the dust formation rate slowed. Plausible causes for the recent brightening involve changes in the stellar wind and its emergent radiation field, which require some change in the star itself. A decrease in the mass-loss rate was proposed (Mehner et al. 2010). Kashi \& Soker (2009a), Mehner et al. (2010), and Corcoran et al. (2010) also inferred a drop in $\eta$ Car's mass-loss rate based on $\eta$ Car's $\mathrm{X}$-ray light curve.

Observations in the far-IR reinforce the idea of an altered stellar system. Gaczkowski et al. (2013) found that $\eta$ Car's 70-500 $\mu \mathrm{m}$ fluxes in 2010 are about a factor of 2 lower than expected from observations obtained 15 years earlier. They argue that the expansion of the Homunculus can only account for a $20 \%$ drop in the far-IR radiation and propose dynamical changes in the circumstellar dust around $\eta$ Car. However, Gomez et al. (2010) found that the flux at $870 \mu \mathrm{m}$, a wavelength region dominated by free-free emission from the ionized wind, was a factor of 3 higher in 2007 than in 1998, which can be primarily accounted for by the cyclical $5.5 \mathrm{yr}$ variations. Radio monitoring by White (priv. comm) ${ }^{4}$. shows that the radio flux at $8.6 \mathrm{GHz}$ $(3.5 \mathrm{~cm})$ in 2006 was around $3 \mathrm{Jy}$, three times more than in 1998. However, the peak flux in cycle 1998-2003.5 was only at $2 \mathrm{Jy}$, supporting a discontinuity around 1998 in the radio as well.

Eta Car shows an intriguing color evolution in the near-IR that appears to be triggered close to periastron. The color $H-K$ progressed to the blue since the beginning of the near-IR observing record in the early 1970s. Figure 2 shows that this blueward evolution in $H-K$ occurred discontinuously within a short time span of only a few weeks close to the periastron passages. Before $1998, J-H$ was basically constant around a value of about 1 mag but has been evolving to the blue since then.

The colors corrected for the long-term brightening trend (see Fig. 4 and its discussion in Sect. 3) suggest that we may be witnessing a temperature change in the extended tail of $\eta$ Car's wind from $3500 \mathrm{~K}$ to $6000 \mathrm{~K}$ as the optically thick near-IR emission region is moving closer to the star and/or the wind temperature is increasing. Support for this idea comes from van Boekel et al. (2003) and Weigelt et al. (2007). Van Boekel et al. (2003) estimated from interferometric VLTI/VINCI observations in 2002 and 2003 that half of the $K$-band flux comes from within $11 \mathrm{AU}$ and estimated a minimum blackbody temperature of $2300 \mathrm{~K}$ concluding that they have spatially resolved the ionized stellar wind. Using interferometric VLTI/AMBER observations taken in 2004/2005, Weigelt et al. (2007) found an image diameter of the $K$-band continuum corresponding to about $5 \mathrm{AU}$, where temperatures of less than $8000 \mathrm{~K}$ are expected.

\subsection{Possible scenarios for the observed changes in $\eta$ Car}

It is highly improbable that the process of dust dissipation by the expanding Homunculus nebula is the sole cause for the observations described above. To explain the recent magnitude and color trends, a second process needs to be invoked. This second process can either be related to bremsstrahlung emission or to hot dust, and is geometrically distinct from the dust clearance process occurring within the Homunculus nebula. The dramatic change in $\eta$ Car's light curves in 1998 and the correlation of the discontinuous near-IR color evolution with periastron passages suggest that the close approach of the companion star is one of the main drivers for the observed changes. It appears conceivable that the close approach of the companion induces alterations in $\eta$ Car's atmosphere and its close environment. The long-term color changes support a change in the size/temperature of the optically thick wind, although a smaller quantity of warm dust can be considered a viable alternative. We will evaluate two scenarios that may not be exclusive and may also not be the only conceivable ones. For the discussion of the long-term evolution here, we do not consider the existence of optically thin free-free emission and its variability, which may drive the cyclic variability.

\section{Scenario I: Homunculus dust dissipation + bremsstrahlung emission.}

For following reasons we suggest that the preferred mechanism is a combination of thinning dust as the Homunculus nebula expands in combination with variability in optically thick bremsstrahlung emission.

1. Photometric variability occurs simultaneously across $J H K L$.

2. Color evidence of an increased temperature.

3. Eta Car's visual extinction is $A_{V} \sim 6-7$ mag (Hillier et al. 2006) and small (<1-2 mag) at $L$. Thus, the relative fluxes

4 See http://www.astro.umd.edu/ white/images/eta_time_ full. html and White et al. (2005). 
between $V(\approx 4.5 \mathrm{mag}$ in 2013) and $L(\approx-1.6 \mathrm{mag}$ in 2013$)$ are consistent without invoking a large dust emission component in $L$.

Dust production in a wind-wind collision region as observed in Wolf-Rayet (WR) stars does not work well for $\eta$ Car. Eta Car is more luminous than WR stars and even with an order of magnitude or two denser wind, its UV radiation will permeate through its wind. There are also a supposedly hot $\left(T_{\mathrm{eff}} \sim 37000 \mathrm{~K}\right)$ companion and colliding wind shocks as additional sources of ionizing photons and energy. It is thus unlikely that dust will form within $150 \mathrm{AU}$ as the local thermal environment is too harsh for dust formation ( $T_{\mathrm{eff}} \sim 1500 \mathrm{~K}$ at $\left.150 \mathrm{AU}\right)$. It is likely that the dust-free region around $\eta$ Car extends out to 200$300 \mathrm{AU}$, which is also inferred from high-angular resolution imaging (see Chesneau et al. 2005). Considering that the significantly dense part of the wind-collision region is contained within $100 \mathrm{AU}$, a rapid dust formation (on a timescale of days to months) will probably not occur. In addition, unlike dust formation in WR stars (Williams et al. 2013), the largest amplitudes of variability in the near-IR of $\eta$ Car are found in the $J$ rather than in $L$ band.

If we assume the near-IR emission is dominated by optically thick plasma, then the observed drop in $L$-band flux below the long-term trend could have been caused by a decreasing effective radius. Here effective radius means the radius at which the optical depth of the plasma changes from optically thick to optically thin. A smaller effective radius could have resulted from a lower mass-loss rate, leading to an increased temperature at the effective radius. This is accounted for by the increased $J$ and $H$ emission above the long-term trend. The $K$ band around $2.2 \mu \mathrm{m}$ may be the pivotal wavelength around which the bound-free and freefree emission component hinges: the $K$-band flux decreases because the emitting volume becomes smaller, but this decrease is compensated for by the temperature increase. Thus the $K$-band flux is almost constant (when corrected for the long-term brightening trend associated with the expanding Homunculus nebula).

We can also speculate on the physical causes of a possible change in mass-loss rate and the importance of the periastron passages. A possible hypothesis for a physical cause is angular momentum transfer between the two stars at periastron. At the point of the closest approach angular momentum could be transferred onto the primary through tidal torques that spin up $\eta$ Car's atmosphere (Davidson 1997). Incidentally, $\eta$ Car's rotational velocity is most likely close to its critical velocity now. Groh et al. (2010) found in VLTI/VINCI and AMBER observations that $\eta$ Car's rotation speed is $80-90 \%$ of its critical rotation speed $^{5}$. The angular momentum transfer scenario appears conceivable since $\eta$ Car's rotational velocity is probably close to the orbital velocity of the companion star, i.e., $v_{\text {rot }} \sim 0.8-0.9 \times v_{\text {crit }} \sim$ $240-270 \mathrm{~km} \mathrm{~s}^{-1}$, whereas $v_{\text {orb }} \sim 250-300 \mathrm{~km} \mathrm{~s}^{-1}$ (with a companion mass of $\sim 40-50 M_{\odot}$ and an orbit eccentricity of 0.8 or higher). Eta Car may have been approaching its critical velocity through an impulsive increase of $v_{\text {rot }}$ at every periastron. The increase also occurred prior to 1998 , but the rotational speed then may not have been close enough to its critical limit to induce the more dramatic changes observed afterward. This scenario could have been potentially enhanced, especially after 1998, if the star had expanded because of an internal instability (Davidson 1997). However, the nature of this internal instability is not clear to the authors. A tidal spin-up of the star may be sufficient to trigger

\footnotetext{
5 However, Groh et al. (2010) note that the primary wind could be sufficiently disturbed by the companion to mimic the effects of fast rotation.
}

some kind of rotational instability and it may not be necessary to invoke an additional internal instability.

Owocki (2005) suggested that the rapid (near-critical) stellar rotation of $\eta$ Car induces an equatorial gravity darkening. While the radiative flux itself scales in proportion to the effective surface gravity, the mass flux scales directly with effective gravity, i.e., it is (contrary to intuitive expectations) at minimum near the equator and at maximum near the poles (Owocki et al. 1996). It may be that the companion star spins up $\eta$ Car incrementally on a regular basis (in addition to any spinning mechanism that may be present in LBVs). Its rotational velocity is approaching its critical velocity, leading to lower effective gravity at the equator, and thus reducing the mass-loss rate there ${ }^{6}$. In view of this scenario, the $J H K$ excess emission during periastron passages can be explained by invoking a disruption of the primary wind, effectively mimicking a decrease in mass-loss rate (i.e., hotter zones of the optically thick wind are exposed; see Madura et al. 2012a and Sect. 4.2).

In a series of papers Soker et al. have discussed an accretion model for $\eta$ Car that argues for the opposite effect from the one we just stated (see, e.g., Soker 2005; Kashi \& Soker 2008a; Kashi \& Soker 2009b). In their scenario, the companion accretes mass from $\eta$ Car during periastron. In this case angular momentum from $\eta$ Car is transferred onto the companion, resulting in a slowing of its stellar rotation. However, the net change in angular momentum of $\eta$ Car depends on the mass fraction of the wind accreted onto the companion. The extended part of $\eta$ Car's wind only carries a negligible amount of the star's total mass, so there may be a net gain in the angular momentum upon periastron passage.

Scenario II: Homunculus dust dissipation + hot dust formation.

The results in the near- to far-IR suggest that the inner dust envelope, enshrouding the central star, is opening up. A larger fraction of the stellar UV to near-IR radiation, which was previously absorbed within the inner dust envelope, is leaving the system, leading to less thermal dust emission at far-IR wavelengths and increased flux at wavelengths dominated by $\eta$ Car's stellar wind.

In principle, the variations and post-1998 behavior could be explained with a scenario of dust removal at periastron and dust formation/recovery at apastron, but there are caveats related to the dust formation environment and the dust formation timescale. From the decreasing $K$ - and $L$-band flux below the long-term trend in the last few years (although the effect on the light curve appears to be minute) we may infer that the amount of hot dust ( $\left.T_{\text {dust }} \sim 1000-1500 \mathrm{~K}\right)$ must have decreased since 1998 , lowering the total extinction and allowing more $J H$ flux to escape. As this is triggered at periastron, the host dust needs to be cleared at periastron, where a wind-collision dust production would be most effective. Prior to 1998, at post-periastron, the hot dust must have newly formed in the course of about one year as the near-IR flux levels are observed to revert to the pre-periastron state and this newly formed hot dust needs to be homogeneously distributed throughout the environment quickly after periastron passage. After 1998, the hot dust does not reappear or at least appears in much smaller quantities. This could be explained with a change in the wind-collision properties, e.g., different densities. If so, then we can assume that different densities occur in the

\footnotetext{
6 Koenigsberger et al. (2001) argued that angular momentum transfer from the companion briefly enhances $\eta$ Car's mass-loss rate during periastron. However, this effect would only occur briefly at the point of closest approach.
} 
primary's wind post-1998 and we arrive at the same conclusion as in Scenario I, namely that $\eta$ Car's mass-loss rate must have decreased.

In a slightly different scenario, we may assume that the hot dust is formed in the primary wind at $R_{\text {subl }} \sim 150 \mathrm{AU}$, but then the question remains why the dust stopped forming after 1998. We note that in Scenario II reasoning along the lines of dust in supergiant B[e] stars (gravity darkening, dense equatorial outflow with dust formation) is hard to uphold, because we need the hot dust to be spherically distributed or at least to be present in our line of sight.

\subsection{Excess emission around the periastron passages}

Eta Car's JHKL light curves show excess emission correlated with the times of the periastron passages. Madura et al. (2012a) proposed a model where the companion introduces a wind cavity in $\eta$ Car's dense wind. This cavity leads to increased radiation from the deeper layers of $\eta$ Car's wind close to periastron. They were able to replicate the light curves around periastron including the excess brightness and the miminum.

Alternatively, Kashi \& Soker (2008a) and Smith (2010) proposed the formation of hot dust $\left(T_{\text {dust }} \sim 1500 \mathrm{~K}\right)$ in the colliding winds to explain the increased near-IR flux around periastron. Falceta-Gonçalves et al. (2005) also showed that conditions in their model are favorable for grain formation. However, it is difficult to sustain a large amount of dust at $T_{\text {dust }} \sim 1500 \mathrm{~K}$ in the harsh environment around $\eta$ Car. Whitelock et al. (2004) rejected an orbital cycle of grain growth and destruction since more dramatic variations in the $L$ band than observed would be expected. Chesneau et al. (2005) found a dust-free area with a radius of 230-350 AU around $\eta$ Car using near-IR high-angular resolution (60 mas resolution) VLT/NACO images. Other explanations for the excess emission at periastron include S Doradus-type variations (Whitelock et al. 1994; Sterken et al. 1999), enhanced freefree emission as newly ejected mass joins the material around $\eta$ Car (Feast et al. 2001), and expansion of the primary due to tidal forces (van Genderen et al. 2006). It is also possible to envisage the excess emission at periastron as a consequence of grain destruction. Increased UV radiation from deeper layers of $\eta$ Car's wind close to periastron may progressively heat large amounts of dust particles up to sublimation temperature of about $1500 \mathrm{~K}$.

\section{Conclusion}

We presented JHKL photometry of $\eta$ Car obtained since 2004 and compared the post-1998 data to the historical record from 1972-1998. We find that the near-IR light curves considerably altered their long-term behavior around 1998 with respect to the general trend from before 1998. The change is most pronounced in $J$ and $H$, where excess emission above the long-term trend is observed. The near-IR data also show strong episodic and long-term color variations. Sudden near-IR color changes appear close to periastron passages. While the pre-1998 colors were practically unchanged when corrected for the long-term brightening trends (which we associate with the expansion of the Homunculus nebula), the post-1998 colors evolve perceptibly toward the blue. The observed changes are inconsistent with a gradual dissipation of dust located in the Homunculus nebula as the sole cause and we discuss other processes that could explain the observations.

We argue that the changing near-IR emission is plausibly caused by variability in optically thick bremsstrahlung emission.
This is supported both by the discontinuity in the long-term trend since 1998 and the shorter term variations related to the binary orbit. We note that the existence of optically thin freefree emission components is also likely, which may contribute to the cyclic variation of colors, especially at the time of periastron passage. We show by means of color-color analysis that the variability may imply that a thermal component, presumably the stellar wind, has become hotter and/or the boundary radius between optically thick and optically thin emission has become smaller. This suggestion is consistent with recent changes in $\eta$ Car's UV, optical, and X-ray light curves and its optical stellar wind features. These observational findings all support an interpretation in terms of a decrease in $\eta$ Car's mass-loss rate resulting in more ionizing photons permeating its stellar wind.

The periastron passages, especially the one in 1998, may be inferred to be the driving force behind the observed quasiperiodic changes related to the orbital period. The fine temporal sampling of the near-IR radiation strongly suggests that periastron passages coincide with discontinuities in the long-term trend and therefore related to a persistent change in the system. This view is also supported by the spectroscopic record. As a possible physical cause we propose that angular momentum transfer between the two stars at periastron may be altering $\eta$ Car's stellar rotation and then in turn its mass-loss rate. This may imply that $\eta$ Car is becoming more unstable as it nears its critical rotation limit, alluding to the possibility of another eruptive phase.

Evidence is increasing that the periastron passages play an important role in the evolution of the system. Further modeling of the interaction of the companion with material close to $\eta$ Car and with its wind during periastron is required to disentangle the various physical processes. From an observational point, nearIR spectral monitoring is needed to provide information on the variability of the HI and He I emission lines and how they influence the observed photometry. Unfortunately, no far-UV data has been obtained since 2003 but UV observations of $\eta$ Car with $H S T$ within the next year will determine if a drop in mass-loss rate occurred. Observations at all wavelengths throughout the next periastron in 2014.5 will be essential. The structure of $\eta$ Car is complex and the interpretation of the near-IR data is thus difficult. Nevertheless, important evidence for the evolution of this system can be obtained from the integrated near-IR photometry as presented in this paper.

Acknowledgements. This paper is based on observations made at the South African Astronomical Observatory (SAAO). A.M. was co-funded under the Marie Curie Actions of the European Commission (FP7-COFUND). K.I. was supported by the Global Center of Excellence Program of Nagoya University from the Japan Society for the Promotion of Science and the Ministry of Education, Culture, Sports, Science and Technology. PAW and MWF acknowledge the receipt of a research grant from the South African National Research Foundation (NRF). We thank Jose Groh, Mikio Kurita, Kris Davidson, and Stan Owocki for valuable discussions.

\section{References}

Allen, D. A. 1989, MNRAS, 241, 195

Carter, B. S. 1990, MNRAS, 242, 1

Chesneau, O., Min, M., Herbst, T., et al. 2005, A\&A, 435, 1043

Corcoran, M. F., Hamaguchi, K., Pittard, J. M., et al. 2010, ApJ, 725, 1528

Damineli, A. 1996, ApJ, 460, L49

Damineli, A., Kaufer, A., Wolf, B., et al. 2000, ApJ, 528, L101

Davidson, K. 1971, MNRAS, 154, 415

Davidson, K. 1997, New Astron., 2, 387

Davidson, K., \& Humphreys, R. M. 1997, ARA\&A, 35, 1 
Davidson, K., \& Humphreys, R. M. 2012, Eta Carinae and the Supernova Impostors (Springer Science + Business Media), Astrophys. Space Sci. Libr., 384

Davidson, K., Ebbets, D., Weigelt, G., et al. 1995, AJ, 109, 1784

Davidson, K., Gull, T. R., Humphreys, R. M., et al. 1999, AJ, 118, 1777

Davidson, K., Ishibashi, K., Gull, T. R., Humphreys, R. M., \& Smith, N. 2000, ApJ, 530, L107

Davidson, K., Martin, J., Humphreys, R. M., et al. 2005, AJ, 129, 900

Falceta-Gonçalves, D., Jatenco-Pereira, V., \& Abraham, Z. 2005, MNRAS, 357, 895

Feast, M., Whitelock, P., \& Marang, F. 2001, MNRAS, 322, 741

Gaczkowski, B., Preibisch, T., Ratzka, T., et al. 2013, A\&A, 549, A67

Gehrz, R. D., Ney, E. P., Becklin, E. E., \& Neugebauer, G. 1973, Astrophys. Lett., 13, 89

Glass, I. S., \& Nagata, T. 2000, Monthly Notes Astron. Soc. South. Africa, 59, 110

Gomez, H. L., Vlahakis, C., Stretch, C. M., et al. 2010, MNRAS, 401, L48

Groh, J. H., Madura, T. I., Owocki, S. P., Hillier, D. J., \& Weigelt, G. 2010, ApJ, 716, L223

Groh, J. H., Hillier, D. J., Madura, T. I., \& Weigelt, G. 2012a, MNRAS, 423, 1623

Groh, J. H., Madura, T. I., Hillier, D. J., Kruip, C. J. H., \& Weigelt, G. 2012b, ApJ, 759, L2

Hillier, D. J., Gull, T., Nielsen, K., et al. 2006, ApJ, 642, 1098

Humphreys, R., \& Stanek, K. 2005, The Fate of the Most Massive Stars. ASP Conf. Ser., 332

Kashi, A., \& Soker, N. 2008a, NewA, 13, 569

Kashi, A., \& Soker, N. 2008b, MNRAS, 390, 1751

Kashi, A., \& Soker, N. 2009a, ApJ, 701, L59

Kashi, A., \& Soker, N. 2009b, New Astron., 14, 11

Koenigsberger, G., Cervantes, F., Moreno, E., \& Georgiev, L. 2001, in The Seventh Texas-Mexico Conference on Astrophysics: Flows, Blows and Glows, eds. J. Cantó, \& L. F. Rodríguez, Rev. Mex. Astron. Astrofis. Conf. Ser., 10, 199

Madura, T. I., Gull, T. R., Groh, J. H., et al. 2012a, in Proceedings of a Scientific Meeting in Honor of Anthony F. J. Moffat held at Auberge du Lac Taureau, St-Michel-Des-Saints, Québec, Canada, held 11-15 July 2011, eds. L. Drissen, C. Rubert, N. St-Louis, \& A. F. J. Moffat, ASP Conf. Ser., 465,313

Madura, T. I., Gull, T. R., Owocki, S. P., et al. 2012b, MNRAS, 420, 2064

Madura, T. I., Gull, T. R., Okazaki, A. T., et al. 2013, MNRAS, 436, 3820

Mehner, A., Davidson, K., Humphreys, R. M., et al. 2010, ApJ, 717, L22
Mehner, A., Davidson, K., Martin, J. C., et al. 2011, ApJ, 740, 80

Mehner, A., Davidson, K., Humphreys, R. M., et al. 2012, ApJ, 751, 73

Morse, J. A., Humphreys, R. M., \& Damineli, A., 1999, Eta Carinae At The Millennium. ASP Conf. Ser., 179

Nagashima, C., Nagayama, T., Nakajima, Y., et al. 1999, Proc. Star Formation 1999, ed. T. Nakamoto, 397

Nagayama, T., Nagashima, C., Nakajima, Y., et al. 2003, in Instrument Design and Performance for Optical/Infrared Ground-based Telescopes, eds. M. Iye, \& A. F. M. Moorwood, Proc. SPIE, 4841, 459

Okazaki, A. T., Owocki, S. P., Russell, C. M. P., \& Corcoran, M. F. 2008, MNRAS, 388, L39

Owocki, S. 2005, in The Fate of the Most Massive Stars, eds. R. Humphreys, \& K. Stanek, ASP Conf. Ser., 332, 169

Owocki, S. P., Cranmer, S. R., \& Gayley, K. G. 1996, ApJ, 472, L115

Smith, N. 2010, MNRAS, 402, 145

Smith, N., \& Gehrz, R. D. 2000, ApJ, 529, L99

Smith, N., Gehrz, R. D., Hinz, P. M., et al. 2003, AJ, 125, 1458

Soker, N. 2005, ApJ, 635, 540

Sterken, C., Freyhammer, L., Arentoft, T., \& van Genderen, A. M. 1999, A\&A, 346, L33

van Boekel, R., Kervella, P., Schöller, M., et al. 2003, A\&A, 410, L37

van Genderen, A. M., \& Sterken, C. 2004, A\&A, 423, L1

van Genderen, A. M., de Groot, M. J. H., \& The, P. S. 1994, A\&A, 283, 89

van Genderen, A. M., Sterken, C., Allen, W. H., \& Walker, W. S. G. 2006, J. Astron. Data, 12, 3

Weigelt, G., \& Ebersberger, J. 1986, A\&A, 163, L5

Weigelt, G., \& Kraus, S. 2012, in Eta Carinae and the Supernova Impostors, eds. K. Davidson, \& R. M. Humphreys, Astrophys. Space Sci. Lib., 384, 129

Weigelt, G., Kraus, S., Driebe, T., et al. 2007, A\&A, 464, 87

White, S. M., Duncan, R. A., Chapman, J. M., \& Koribalski, B. 2005, in The Fate of the Most Massive Stars, eds. R. Humphreys, \& K. Stanek, ASP Conf. Ser., 332, 126

Whitelock, P. A. 2005, in The Fate of the Most Massive Stars, eds. R. Humphreys, \& K. Stanek, ASP Conf. Ser., 332, 115

Whitelock, P. A., Feast, M. W., Carter, B. S., Roberts, G., \& Glass, I. S. 1983, MNRAS, 203, 385

Whitelock, P. A., Feast, M. W., Koen, C., Roberts, G., \& Carter, B. S. 1994 MNRAS, 270, 364

Whitelock, P. A., Feast, M. W., Marang, F., \& Breedt, E. 2004, MNRAS, 352, 447

Williams, P. M., van der Hucht, K. A., van Wyk, F., et al. 2013, MNRAS, 429, 494 